\title{
CHARACTERIZATION OF OXIDE SUPPORTED RHODIUM NANOCRYSTALS: HABIT, SURFACE STRUCTURE AND CATALYTIC ACTIVITY
}

\author{
G. Rupprechter, K. Hayek, and H. Hofmeister ${ }^{1}$ \\ Institute of Physical Chemistry, Leopold-Franzens-Universität Innsbruck, \\ Innrain 52a, A-6020 Innsbruck, Austria \\ ${ }^{1}$ Max Planck Institute of Microstructure Physics, Weinberg 2, \\ D-06120 Halle, Germany
}

\begin{abstract}
Well-facetted $R$ h nanocrystals, epitaxially grown on $\mathrm{NaCl}$ substrates and supported by amorphous alumina, were selected to study the effect of annealing in $\mathrm{O}_{2}$ and $\mathrm{H}_{2}$ on the particle microstructure and catalytic properties. The habit and the surface composition of the crystallites were determined by high resolution electron microscopy assisted by image contrast simulation, and by electron diffraction. Their catalytic performance in the hydrogenolysis of alkanes was tested by microreactor kinetics. Model catalysts with high turnover frequencies mainly exhibited Rh particles with disordered surface structures (e.g. polycrystalline Rh particles or sandwich structures of $\mathrm{Rh}$ and $\mathrm{Rh}_{2} \mathrm{O}_{3}$ ), while smooth low-index facets dominated in less active catalysts. The results confirm that low-coordinated sites are preferred for hydrogenolysis.

(C) 1997 Acta Metallurgica Inc.
\end{abstract}

\section{INTRODUCTION}

The complexity of industrial supported catalysts consisting of randomly oriented and irregularly shaped metal particles on high surface area porous supports generally prevents their unambiguous characterization by electron microscopy and by uhv-based surface science techniques. Therefore, catalytic model systems are frequently invoked in studies of structure sensitive reactions and mainly two different approaches have been attempted in the past: Single crystal metal surfaces in combination with uhv high pressure cells (1) and small metal particles supported on suitable model supports (2). In this respect, well-facetted noble metal nanocrystals supported on thin films of amorphous oxides provide an appropriate model which is particularly suited for high resolution electron microscopy (HREM) (3). In this communication we present an attempt to correlate the surface structure, respectively the surface composition of nanometersized Rh particles with their catalytic properties.

The rates and product selectivities of supported $\mathrm{Rh}$ in the hydrogenolysis of alkanes depend sensitively on the treatment applied to activate or to rejuvenate the catalyst $(4,5,6)$. Catalyst activation generally involves annealing in oxygen and subsequently in hydrogen and does not only burn off impurities from catalytically active surfaces but also leads to distinct microstructural changes of the metal particles. These structural alterations of Rh nanocrystals will be examined by electron microscopy and their influence on the catalytic performance will be discussed. 


\section{HABIT AND SURFACE STRUCTURE OF AS-PREPARED RH NANOCRYSTALS}

Nanosized $\mathrm{Rh}$ particles were grown epitaxially on $\mathrm{NaCl}(001)$ surfaces by physical vapour deposition at substrate temperatures between 600 and $650 \mathrm{~K}$ and a base pressure of $1 \times 10^{-5} \mathrm{~Pa}$. The metal deposition was accomplished by electron beam evaporation of a $\mathrm{Rh}$ rod at a flux of $1.2 \times 10^{13}$ atoms cm-2 $\mathrm{s}^{-1}$ up to a nominal layer thickness of $0.8 \mathrm{~nm}$. The samples were cooled to room temperature and subsequently covered with $25 \mathrm{~nm}$ of amorphous alumina by reactive evaporation of $\mathrm{Al}$ in $1 \times 10^{-2} \mathrm{~Pa} \mathrm{O}_{2}$. For electron microscopy examination the $\mathrm{Rh} /$ alumina films were floated from the substrate in water, rinsed and mounted on gold grids. Fourier transforms of digitized HREM images were utilized to determine interplanar angles and spacings. Two types of $\mathrm{NaCl}$ substrates were used in parallel, i.e. vacuum-cleaved $\mathrm{NaCl}(001)$ single crystal faces $\left(0.25 \mathrm{~cm}^{2}\right)$ and in-situ deposited $\mathrm{NaCl}$ films $\left(100 \mathrm{~cm}^{2}\right)$ which provide sufficient area for kinetic measurements in a microreactor. Further experimental details are given elsewhere (3).

The epitaxial growth of $\mathrm{Rh}$ on $\mathrm{NaCl}$, as evident from electron diffraction, leads to the formation of a variety of polyhedral particles about 3 to $10 \mathrm{~nm}$ in size (Fig. la,c). Most of them exhibit [001] or [011] zone axes parallel to the electron beam and the examination of their morphology, orientation and microstructure by HREM and weak-beam dark-field (WBDF) imaging is therefore considerably facilitated. Particles with square outlines were most abundant and according to HREM (Fig. 1b) and WBDF (3) they are of half octahedral shape with (001) base planes and $\{111\}$ side faces. A detailed analysis of the other types of particle shapes observed, revealed the presence of $(001)$ oriented pseudo-pentagonal pyramids, (011) half tetrahedra, $(011)$ cuboctahedra, and of a variety of twinned and multiply-twinned particles (7). Half tetrahedra and cuboctahedra in (011) orientation exhibit $\{111\}$ and $\{100\}$ bounding faces parallel to the electron beam and allow the surface structure of these edge-on facets to be recorded. Mainly smooth facets were observed and only a few surface steps were apparent.

Like any other freshly prepared catalyst, the epitaxial model catalyst films exhibited only very low catalytic activity in the as-prepared state. An activity maximum in the hydrogenolysis of alkanes was achieved only after oxidation in $10^{5} \mathrm{~Pa} \mathrm{O}_{2}$ at $675 \mathrm{~K}(30-120 \mathrm{~min})$ followed by a low temperature reduction in $\mathrm{H}_{2}$ at $525 \mathrm{~K}$ ( $30 \mathrm{~min}$; LTR). However, the turnover frequency decreased by one to several orders of magnitude if the oxidation temperature was decreased to $575 \mathrm{~K}$ or if the reduction temperature was increased to $725 \mathrm{~K}$ (high temperature reduction; HTR) (6).
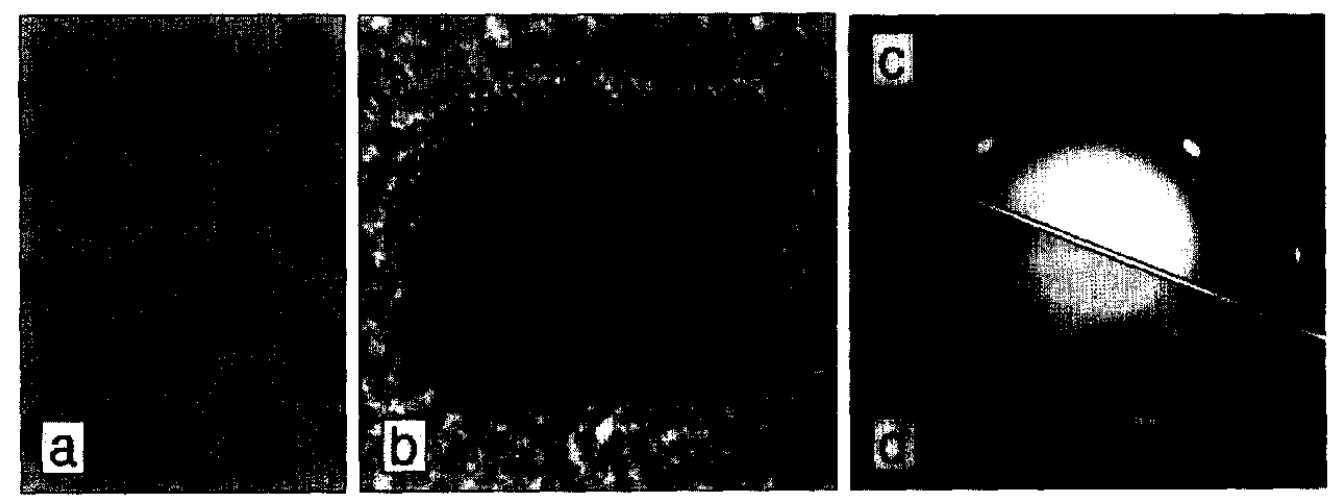

Fig. 1: (a) Electron micrograph of an epitaxially grown $\mathrm{Rh} / \mathrm{Al}_{2} \mathrm{O}_{3}$ model catalyst; (b) HREM image of a Rh pyramid viewed along [001]; electron diffraction pattern "as-prepared" (c) and after oxidation at $725 \mathrm{~K}, 120 \mathrm{~min}$ (d); oxide spots are marked by arrows. 


\section{MICROSTRUCTURAL CHANGES UPON ANNEALING IN $\mathrm{O}_{2}$ AND $\mathrm{H}_{2}$}

After heating in $\mathrm{O}_{2}$ at $575 \mathrm{~K}$ oxide lattice plane fringes were observed at the edges of pyramidal particles (Fig. 2a). According to HREM the oxide skin appeared to be restricted to the particle edges but more probably the thick metal core prevented imaging of the total surface oxide. Upon oxidation at 675 to $725 \mathrm{~K}$ the oxide shells increased in thickness but apparently were still limited to the particle periphery. An epitaxial relationship between the metal (Fig. 1c) and the oxide (Fig. 1d) was revealed by electron diffraction and according to the observed spacings and symmetry the oxide is hexagonal $\alpha-\mathrm{Rh}_{2} \mathrm{O}_{3}$. The orientation relationship was determined to be (035) $\mathrm{Rh}_{2} \mathrm{O}_{3} / /(001) \mathrm{Rh}$, or more precisely [031] $\mathrm{Rh}_{2} \mathrm{O}_{3} / /$ [001] $\mathrm{Rh}$ (zone axes) and [100] $\mathrm{Rh}_{2} \mathrm{O}_{3}$ // [110] Rh (crystallographic directions). Taking this into account a model structure of the oxidized particles for image contrast calculations could be set up by combining supercells of Rh and $\mathrm{Rh}_{2} \mathrm{O}_{3}(6)$. The ratio between the thickness of the metal and of the oxide was varied in an number of defocus series and it could be shown that the oxide must be 3 to 5 times thicker than the metal in order to be imaged by HREM (Fig. 3). Under the given conditions this can only be achieved at the particle edges, in good agreement with the observed images. Upon LTR of particles oxidized at $575 \mathrm{~K}$ the surface oxide is decomposed and the initial morphology is regained.

Particles which were previously oxidized at $675 \mathrm{~K}$ and subsequently reduced at $525 \mathrm{~K}$ (LTR) exhibited mainly polycrystalline structures, as evident from HREM and WBDF. In Fig. 2b a particle was selected for illustration, which still shows lattice resolution on most parts. Besides lattice plane fringes of 0.22 and $0.19 \mathrm{~nm}$ spacing, corresponding to $\mathrm{Rh}\{111\}$ and $\{100\}$, fringe spacings of 0.24 and $0.18 \mathrm{~nm}$ were detected. These spacings correspond neither to $\mathrm{Rh}$ or to $\mathrm{Rh}_{2} \mathrm{O}_{3}$ and are probably due to interference effects resulting from the superposition of different crystalline materials: Oxidation at $675 \mathrm{~K}$ had formed an oxide envelope around the metal cores but had not completely oxidized the Rh particles. Upon LTR islands of metal are formed on the oxide surface, and if this reduction is incomplete rather complex sandwich structures such as $\mathrm{Rh} / \mathrm{Rh}_{2} \mathrm{O}_{3}$ / Rh will probably be created. This model is supported by calculated HREM images revealing that the combination of oxide and metal layers in a thickness ratio of 2:1 leads to lattice fringe spacings which cannot be attributed to either of these materials (Fig. 3b).

If $\mathrm{Rh}$ particles preoxidized at $675 \mathrm{~K}$ are subjected to HTR, the oxide shells are completely removed and, with the exception of truncations at the corners, the as-grown habits are perfectly re-established.
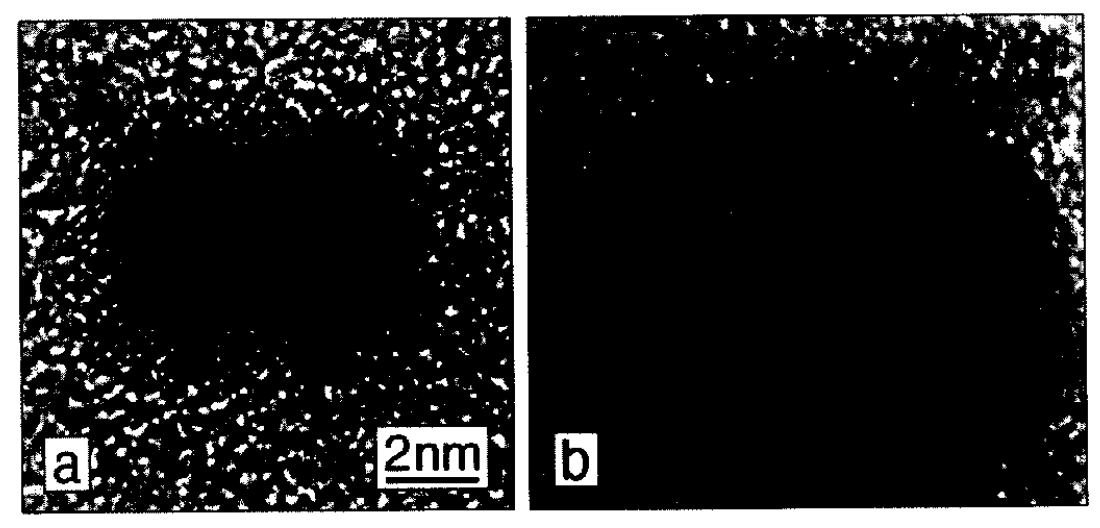

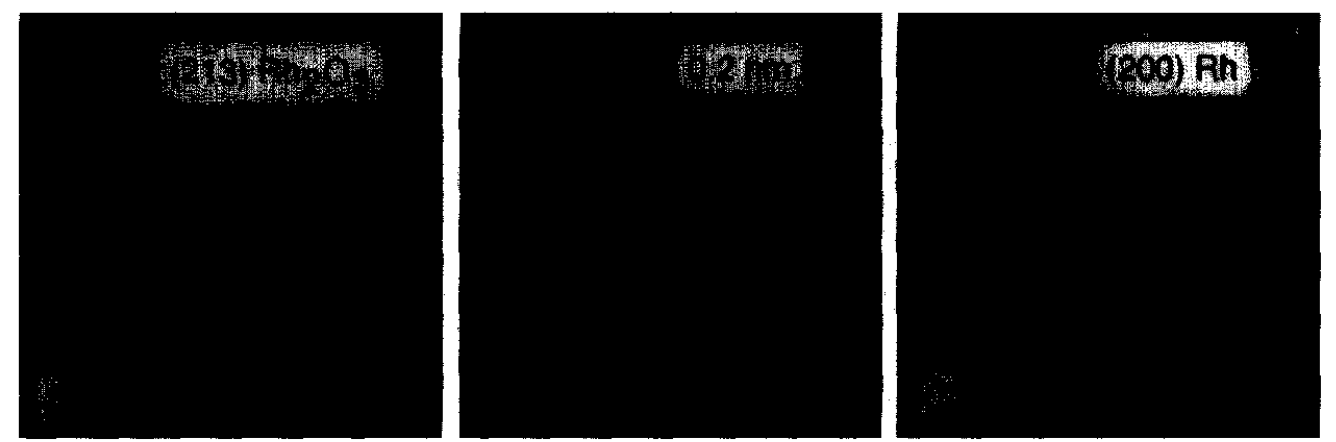

Fig. 3: HREM image contrast calculation of an $\alpha-\mathrm{Rh}_{2} \mathrm{O}_{3}$ overlayer on $\mathrm{Rh}$ for different oxide to metal thickness ratios: (a) 5:1, (b) 2:1, and (c) 1:1 (imaging parameters according to JEM 4000 EX, near Scherzer defocus).

\section{CONCLUSIONS}

The catalytic performance of alumina supported $\mathrm{Rh}$ depends sensitively on the surface structure of the metal particles. From HREM investigations it is clear that as long as the pyramidal habit of the $\mathrm{Rh}$ particles was retained or regained, i.e. as long as mainly low-index facets were exposed, their activity was only moderate. However, an activity maximum was observed after a preoxidation at $675 \mathrm{~K}$ and LTR, which resulted in the formation of polycrystalline $\mathrm{Rh}$ particles, and presumably in the formation of complex sandwich structures. While highcoordination sites dominate on low-index faces (e.g. $\mathrm{C}_{9}$ according to (8)), the polycrystalline particles consist of smaller crystalline domains and expose mainly low-coordinated sites, i.e. their surface is considerably roughened. The observed increased activity of low-coordination sites is in good agreement with studies performed on flat, stepped and kinked noble metal single crystal surfaces in a series of alkane hydrogenolysis reactions where maximum rates were obtained on surfaces containing high concentrations of steps and kinks (e.g. (9)).

\section{REFERENCES}

1. G. A. Somorjai, Surf. Sci. 299/300, 849 (1994).

2. A. K. Datye and D. J. Smith, Catal. Rev. - Sci. Eng. 34, 129 (1992).

3. G. Rupprechter, K. Hayek, L. Rendón and M. José-Yacamán, Thin Solid Films 260 , 148 (1995).

4. L. D. Schmidt and K. R. Krause, Catal. Today 12, 269 (1992).

5. D. Kalakkad, S. L. Anderson, A. D. Logan, J. Peña, E. J. Braunschweig, C. H. F. Peden and A. K. Datye, J. Phys. Chem. 97, 1437 (1993).

6. G. Rupprechter, K. Hayek and H. Hofmeister, J. Catal, in preparation.

7. G. Rupprechter, K. Hayek and H. Hofmeister, Vacuum 46, 1035 (1995).

8. R. van Hardeveld and F. Hartog, Surf. Sci. 15, 189 (1969).

9. S. M. Davis, F. Zaera and G. A. Somorjai, J. Am. Chem. Soc. 104.7453 (1982).

Acknowledgements G.R. is indebted to the Max-Planck-Gesellschaft for a SC fellowship. Helpful discussions with Prof. W. Neumann and Dr. D. Timpel are gratefully acknowledged. 